\title{
Mediatividad, Omni-locutores, y marcadores del discurso. El caso del francés actual: on sait que, on dit que, on prétend que, on raconte que, on admet que
}

Recibido: 14/07/2014

Aceptado: 23/09/2014

\section{RESUMEN:}

Este trabajo se propone estudiar algunas propriedades semántico-pragmáticas de los prefijos verbales on sait que, on dit que, on raconte que, on prétend que, on admet que, en francés actual, y especificar cómo su funcionamiento puede relacionarse con varias temáticas de la pragmasemántica, a saber: los verbos de decir, los marcadores discursivos, la polifonía (especialmente en lo que atañe al concepto de OMNI-locutor o comunidad lingüística), la mediatividad y last but not least los estereotipos. El análisis de los cinco prefijos pone de relieve el valor epistémico propio de cada uno: cada prefijo consiste en un posicionamiento específico del locutor respecto al contenido proposicional.

PALABRAS CLAVE: mediatividad, omni-locutores, marcadores del discurso, modalidades epistémicas, estereotipos, semántica, pragmática.

\section{ABSTRACT:}

This study aims at accounting for some semantic and pragmatic properties of Modern French verbal prefixes on sait que, on dit que, on raconte que, on prétend que, on admet que, and at specifying how their functioning can be related with several topics of pragmasemantics: verbs of saying, discourse markers, generic speaker as figuring a language community, evidentiality and last but not least, linguistic stereotypes. The results of the analysis of these five markers brings out 
their epistemic specificity: each prefix refers to a specific viewpoint of the locutor as far as the truth of the proppositional content is concerned.

KEY WORDS: evidentiality, generic speakers, discourse markers, espitemic modalities, stereotypes, semantics, pragmatics.

\section{Presentación'1.}

Esta pequeña contribución tiene por objeto ofrecer algunas propiedades semántico-pragmáticas de los prefijos verbales on sait que, on dit que, on raconte que, on prétend que, on admet que, en francés actual ${ }^{2}$. Mi interés algo reciente por tales prefijos procede de la observación intuitiva que la función que desempeñan puede relacionarse con varias temáticas de la pragmasemántica, a saber:

a) Los verbos de decir, menos saber. Incluso cuando un saber es o procede de un decir, no se presenta como tal, sino como algo externo ${ }^{3}$.

b) Los marcadores del discurso, ya que son prefijos cuya función se puede tildar de metalingüística: introducen una valoración del contenido oracional externa a la función predicativa ${ }^{4}$.

1 Nuestros más sinceros agradecimientos a: $\mathrm{M}^{\mathrm{a}} \mathrm{L}$. Donaire (Univ. de Oviedo), D. Flament (Univ. de París X), C. Foullioux (Univ. Autónoma de Madrid), M. Djean (Univ. de Zaragoza) por varias sugerencias y críticas; a D. Serrano Dolader y Jé. F. Val Álvaro (Univ. de Zaragoza) por llamar nuestra atención sobre el comportamiento a veces imprevisible de las modalidades espistémicas.

2 Estos prefijos abundan en francés actual, y son de uso cotidiano. Se les llama prefijos verbales porque tienden a independizarse del verbo y formar auténticos marcadores. Por ejemplo, el francés on dirait que significa grosso modo 'por lo visto', tiene poco que ver con decir, y ya no es un condicional pese a las apariencias. Incluso existe la correspondiente forma parentetizada on dirait.

3 Véase por ejemplo el contraste: Eres tú el culpable, en esto estoy de acuerdo con Margarital Digo que eres tú el culpable, en esto estoy de acuerdo con Margarita / ??Sé que eres tú el culpable, en esto estoy de acuerdo con Margarita.

4 Tienen por tanto algo que ver con la integración de las unidades predicativas en el discurso, Véase sobre el tema Zorraquino: 1998, Portolés: 1998a, 1998b. 
c) La polifonía. El locutor de:

(1) Dicen en el pueblo que el caminante volvió.

no es, evidentemente, el 'autor' de el caminante volvió, siendo este el pueblo o una parte no definida del pueblo. En breves términos, reflejan la actitud del locutor hacia el enunciado, en tanto que esa actitud consiste entre otras cosas en situarse dentro de la red de las 'voces' escenificadas en el discurso'.

d) La mediatividad': el locutor de tales prefijos indica la fuente, el origen de la información que transmite el contenido de su enunciado. Ya que todos los verbos aquí estudiados son de tipo 'decir', se trata pues de una mediatividad de mención, citacional. Veremos que en todos los casos los verbos contemplados introducen un valor modal epistémico, y que obviamente no se trata de mención pura.

e) Remiten a un concepto que nos parece sumamente importante, y desempeña un papel central en la teoría que me tiene ocupado últimamente, la teoría de los estereotipos, y es la noción (lingüística) de comunidad lingüística.

f) Veremos al final de esta pequeña contribución, a modo de colofón (el postre del chef), que también tienen alguna conexión con la gnomicidad y la genericidad.

\section{Algunos prolegómenos.}

\subsection{Los verbos de decir.}

Los verbos savoir ('saber'), dire ('decir'), raconter ('contar'), admettre ('admitir, reconocer'), tienen aproximadamente el mismo

5 Desde nuestro punto de vista, las voces son la interpretación en la superficie de las fuentes de los puntos de vista de la estructura profunda. Vid. sobre el tema Anscombre: 2013.

$6 \mathrm{El}$ término evidencialidad se ha tildado de anglicismo y de contrasentido, y con algo de razón. Ya que existe la categoría del mediativo, parece lógico acuñar un término como mediatividad. 
significado que los correspondientes verbos españoles. El caso conflictivo es el verbo prétendre, que coincide con pretender únicamente en el caso de prétendre $\grave{a}^{7}$, y es verbo de decir en todos los demás empleos. Significa aproximadamente 'afirmar sin dar pruebas de ello', y da lugar a los siguientes contrastes con su análogo español:

(2) *Pretende que le robaste la cartera/Il prétend que tu lui as volé son portefeuille.

(3) Pretendo que mi hijo acabe la carrera/*Je prétends que mon fils finisse ses études ${ }^{8}$.

Para evitar problemas de traducción, haremos lo siguiente: dejaremos los prefijos en francés entre corchetes, y pondremos los demás contenidos en español. Lo cual nos llevará a ejemplos con la siguiente forma:

(4) [On dit] en mi pueblo que el caminante volvió.

\subsection{El on francés.}

Sabido es que el francés tiene dos series de pronombres personales: una serie de pronombres tónicos (o fuertes) y facultativos, y una serie de pronombres personales átonos (o débiles) obligatorios al menos cuando no hay ningún sujeto explícito. Entre estos últimos, tiene la lengua francesa un pronombre indefinido de tercera persona, on, que no remite

7 Por ejemplo: François prétend aux plus hauts postes/Francisco pretende los más altos cargos.

8 Sin embargo, se documentan en el habla cotidiana casos como Pretende haber encontrado la solución, siendo la correspondiente subordinada imposible: *Pretende que ha encontrado la solución. No es el único caso de ese tipo, siendo otro el verbo encontrar 'opinar': Lo encuentro muy bueno/*Encuentro que es muy bueno. Por otra parte, el adjetivo pretendido 'presunto', aunque figura en el DRAE, es muy poco frecuente, cuando el francés prétendu 'presunto' es banal y corriente. 
a ninguna entidad específica ${ }^{9}$ en la mayoría de sus empleos ${ }^{10}$. Desempeña muchos papeles, siendo uno de ellos la referencia a una comunidad más o menos amplia, y de contornos borrosos. No existe tal pronombre personal en español, y se suele suplir esta carencia por varios medios, siendo los más frecuentes el plural y las construcciones pasiva y pasiva refleja, lo que queda ilustrado en:

(5) "Se conoce que este muchacho no tiene madre, fíjese usted, solía decir a Barceló..." (C. Ruiz Zafón, La sombra del viento, pág. 53) [On voit bien que ce garçon n’a pas de mère].

(6) «Sabido es que a quien madruga, Dios le ayuda, pero lo suyo es como pedirle una beca, joven,..." (idem, pág. 534) [On sait que l'avenir appartient à celui qui se lève tôt].

(7) «Durante la guerra civil que dicen que hubo no hace mucho en este país, varias personas trataron de cruzar la frontera..." (E. Mendoza, El laberinto de las aceitunas, 1986, Barcelona, Seix Barral, pág. 231) [la guerre civile dont on dit qu'elle eut lieu il n'y a pas si longtemps dans ce pays].

(8) «Se han detectado intentos muy claros de injerencia en dichos proceso..." ( $A B C, 14 / 10 / 2005$, pág. 2) [On a détecté des cas très clairs d'ingérence].

El catalán tiene un pronombre muy parecido al on francés, a saber hom/om, que si bien aún se usa hoy en día, es muy poco frecuente y ha sobrevivido sobre todo en la combinación tothom 'todo el mundo, todos', como en Ho sap tothom, literalmente 'Todo el mundo lo sabe', o sea 'Es del dominio público'.

En el caso de los prefijos aquí estudiados, el on remite siempre a una persona indeterminada, y de alcance impreciso, siendo concretamente uno de los problemas que nos tendrá ocupados

9 Se documenta como persona indeterminada desde el siglo XI.

10 Vid. Leeman: 1991, para los varios usos de on en francés moderno. 
el de deslindar los límites de la comunidad lingüística que evoca cada uno de los prefijos contemplados.

\subsection{La noción de OMNI-locutor.}

Fue acuñado este concepto partiendo del concepto de $\mathrm{ON}$ vérité de Berrendonner: 1981, para dar cuenta de fenómenos vinculados con contenidos presupuestos. El problema radica en el tipo de locutor que interviene en el juego enunciativo, o mejor dicho polifónico. Si bien hay innumerables ejemplos de locutores/voces específicos y determinados, puede también ser el caso que aparezca una voz general y anónima, un OMNI-locutor (fr. ON-locuteur). Los refranes y por lo general las frases sentenciosas son casos muy claros de OMNI-locutores ${ }^{11}$ : traer a colación una frase sentenciosa viene a ser lo mismo que hablar a través de un OMNI-locutor, en este caso el pueblo, la sabiduría popular, el saber del pueblo, el dominio público, etc., en breves términos una comunidad lingüística. Veamos un par de ejemplos:

(8) En todas partes se cuecen habas, como se suele decir.

(9) Cuando el río suena, agua lleva, dicen.

(10) Dice la sabiduría popular que cuando las barbas de tu vecino veas rapar pon las tuyas a remojar (El País, 02/10/99, pág. 2) ${ }^{12}$.

Obsérvese que hay también OMNI-locutores que no son responsables de un decir, sino detentores de un saber. La diferencia

11 Vid. Anscombre: 2010c.

12 Este ejemplo nos fue comunicado por S.Gómez Jordana (Univ. Autónoma de Madrid). Prefijos como como dijo no sé quién, como dijo el otro, como dijo alguien, tendrían que incluirse en la lista de marcadores de OMNI-locutor, ya que desempeñan el mismo papel. He aquí un par de ejemplos: "Más matan los médicos que el verdugo, dijo alguien..." (A. Pérez-Reverte, El oro del rey, Las aventuras del capitán Alatriste IV, Punto de lectura, Madrid, 2003, pág. 177); “¿Te acuerdas de aquello que dijo no sé quién de que el hombre es un lobo para el hombre?..." (C.-J. Cela, Mazurca para dos muertos, Ed. Seix Barral , Barcelona, 1983, pág. 155). 
aparece claramente en las combinaciones con como se suele decir y como sabemos:

(11) (Como sabemos $+{ }^{*}$ como se suele decir), los simios comen plátanos.

(12) (Como sabemos + como se suele decir), la vida da muchas vueltas ${ }^{13}$.

Para Berrendonner, hay una sola entidad OMNI-locutor, que puede incluir o no al locutor, y remite a cualquier "conjunto de individuos" ${ }^{\prime 14}$, siendo más bien confusos según él los contornos de esta tal opinión pública.

Nos proponemos mostrar a través del examen de los marcadores arriba mencionados, que, contrariamente a lo sugerido por Berrendonner, hay varios OMNI-locutores posibles, y que a cada uno corresponde una estrategia enunciativa específica, particularmente en lo tocante al posicionamiento ${ }^{15}$ del locutor respecto al OMNI-locutor.

\section{Los marcadores de decir: algunos elementos de análisis.}

\subsection{Marcadores de decir y comunidad lingüística.}

Examinaremos primero a qué tipo de locutor(es) remite el on presente en los marcadores aquí estudiados, a los que llamaremos

13 Este contraste demuestra que si bien un decir comunitario puede considerarse como un saber, un saber comunitario no puede reducirse a un decir comunitario.

14 ON étant «...l'opinion publique...» (p.41); «...ce ON majuscule...Il s'agit pour moi d'un signifié susceptible de renvoyer déictiquement à n'importe quel ensemble d'individus parlants, de manière parfaitement indéterminée...ON peut inclure, aussi bien que ne pas inclure, le locuteur et/ou le destinataire, et ceci reste affaire de conjecture: les contours de l'opinion publique sont toujours moins nets que ceux des individus...» (p.45); «...ON que j'appelle, faute de mieux, l'opinion publique, parce que son rôle est le plus souvent de dénoter une doxa anonyme...» (Berrendonner: 1981, pág. 58)

15 Inglés commitment, francés prise en charge. Vid. Donaire: 2008. 
marcadores mediativos genéricos ${ }^{16}$.De hecho, la presencia de un on, si bien representa un indicio prometedor, no asegura del todo una interpretación en términos de OMNI-locutor. El on francés, desgraciadamente, es bastante polifacético, y si bien puede remitir a un locutor genérico - una comunidad lingüística indefinida, como en:

(13) [On sait que] a los simios les gustan los plátanos (= 'Sabido es...").

también puede señalar a un locutor específico, perfectamente delimitado:

(14) Nosotros, en casa, [on mange] (='comemos') jabugo cada día.

Hemos entonces de mostrar que los marcadores estudiados funcionan como en (13), y no como en (14). Son dos los argumentos que pueden aducirse en favor de esta primera característica: a) Es posible insertar elementos que implican un origen difuso del contenido proposicional:

(15) $[$ On dit + on raconte + on admet + on prétend + on sait $]$ generalmente que la Biblia tiene varios autores.

(16) [On dit + on raconte] en todas partes que el ministro de Hacienda estaría implicado en el asunto.

(17) $[$ On dit + on raconte + on admet + on prétend + on sait $], y$ por lo visto, es del dominio público, que una huelga general es más que probable.

b) Estos prefijos van muy a menudo acompañados de la designación de una entidad global con poca posibilidad de referencia a individuos concretos:

(18) [On prétend] en los círculos autorizados que el nombre del terrorista es conocido.

(19) [On admet] en el Gobierno que habrá que cambiar la ley.

(20) [On raconte] en la prensa que el policía disparó sin motivo.

16 Son genéricos porque se oponen a los específicos como para él, por mí, según tú, véase Coltier-Dendale: 2004 sobre el tema. 
Veamos un par de casos auténticos:

(21) "Se dice en ciertos círculos políticos que, en algunos casos hay un "tapado" al que se apoya sin notoriedad para no contaminarlo..." ( $A B C, 14 / 10 / 2005$, pág. 3).

(22) "A todo esto, como es sabido, el SPD se queda con la cartera de Exteriores..." ( $A B C, 14 / 10 / 2005$, pág. 7).

Hay más: cuando se trata de un colectivo no genérico sino específico, la pregunta ¿Quién?/¿Quiénes? tiene sentido, cuando ésta no lo tiene, si se trata esta vez de un colectivo genérico. Compárese por ejemplo (23) y (24):

(23) - En casa, [on mange] jabugo cada día. - ¿Y quién come jabugo en tu casa? - Mi padre, mi madre, mis hermanos y yo.

(24) - [On raconte] en la prensa que el policía disparó sin motivo. - *Quién, en la prensa?

En este último caso, la pregunta adecuada sería más bien ¿Dónde, en la prensa? Es una característica general de los OMNI-locutores, como se puede comprobar. En el siguiente ejemplo:

(25) “Igualmente, cediendo a las exigencias del régimen castrista, han aceptado que en la Declaración Final se haga mención al "bloqueo" que no al "embargo" de los Estados Unidos..." (ABC, 14/10/2005, pág. 3).

la pregunta ¿Quién? o ¿Quiénes? sería totalmente inapropiada.

\subsection{Saber fundado y saber alegado.}

Puede atribuirse el origen de un conocimiento dado a un personaje discursivo sin más miramientos, es decir, sin evocar ningún proceso observacional o experimental para afianzar la credibilidad de este saber. Hablaremos entonces de un saber alegado, concepto retomado de Anscombre (2006b). Por el contrario, el mismo saber puede presentarse como basado en un 
proceso objetivo o supuesto tal, de origen esta vez experimental u observacional, o bien deductivo stricto sensu. Hablaremos entonces de un saber fundado. En el caso que nos ocupa, podemos ver que on (dit + raconte + prétend + admet) que remiten a un saber alegado, cuando on sait que remite esta vez a un saber fundado ${ }^{17}$. Véase por ejemplo:

(26) $[$ On dit + on raconte + on prétend + on admet] que Marte no tiene atmósfera, pero faltan pruebas conclusivas.

(27) ??[On sait] que Marte no tiene atmósfera, pero faltan pruebas conclusivas.

En realidad, las cosas son un poco más complicadas, debido al hecho que el origen de un saber puede ser múltiple. En el caso de (27), las únicas pruebas de la falta de atmósfera en Marte son las científicas, y no puede haber otras al menos por el momento. En casos menos tajantes, el saber puede proceder de distintos orígenes, como es el caso de:

(27a) [On sait] que la climatología del planeta está cambiando, pero faltan pruebas conclusivas.

Significa (27a) que el saber evocado no procede por ejemplo de un razonamiento científico, sino de extrapolaciones sobre una base por ejemplo experimental, que no conducen a pruebas auténticas. En cambio, en el momento en que los fundamentos del saber quedan bien especificados, el fenómeno observado en (27) vuelve a producirse:

(27b) ??[On sait], y por motivos científicos, que la climatología del planeta está cambiando, pero faltan pruebas conclusivas.

Una última prueba de la presencia del rasgo [+alegado] en los prefijos on dit que, on raconte que, on prétend que, on admet que, y del rasgo [+fundado] en el caso de on sait que es la posible combina-

17 La formulación correcta sería 'presenta un contenido proposicional como siendo un saber fundado'. 
ción con el condicional de discurso referido, el famoso condicional de rumor ${ }^{18}$ :

(28) $\left[{ }^{*}\right.$ On sait + on dit + on raconte + on prétend + on admet $]$ que estarían teniendo lugar conversaciones entre el Gobierno y los terroristas.

\subsection{Tesis probable, tesis improbable.}

Además de la diferencia entre saber fundado y saber alegado, otra diferencia influye también en el momento de pasar de eventuales datos a la conclusión. Así, tendremos la doble posibilidad:

(29) [On dit + on sait] que la física cuántica va a revolucionar el mundo de la física moderna.

debido a que en (29) pueden verse las consecuencias de una teoría científica como base de una actitud valorativa, o bien de un proceso deductivo. Pero cuando domina el rasgo apreciativo, on sait que resulta menos adecuado, así como on admet que ${ }^{19}$ :

(30) [?On sait + on dit + on raconte + on prétend + ?on admet $]$ que la última novela de Montalbán es verdaderamente una auténtica obra maestra.

¿Cómo puede ser así cuando acabamos de afirmar que on admet que remite a un saber alegado?

Para dar cuenta de este fenómeno, examinaremos primero una serie de contrastes. Veamos por ejemplo:

(31) [??On admet + *on sait] que Cristóbal Colón era genovés, pero no hay nada que apoye esta tesis.

(32) [On admet] que Cristóbal Colón era genovés, y efectivamente, hay varios datos que apoyan esta tesis. ñol.

18 Véanse Haillet; 2002, para el francés, y Casado Velarde; 1995, para el espa-

19 Este último prefijo por motivos que estudiaremos más adelante. 
De lo cual deducimos que on sait que implica un saber totalemente fundado, y on admet que un saber que no es totalmente alegado, ya que es parcialmente fundado como se puede observar en (31) y (32).

Veamos ahora el contraste:

(33) [On sait] que este proyecto no es viable, pero [on dit + on raconte + on prétend $+{ }^{*}$ on admet] que sí lo es.

Daremos cuenta de estos fenómenos mediante una nueva distinción entre tesis probable y tesis improbable. Tesis probable será toda tesis $t$ para la cual existen argumentos $p, q, r$, que permiten sacar la conclusión $t$. Si no hay tales argumentos, o si los hay pero insuficientes, la tesis $t$ será una tesis improbable, como vemos en:

(34)Esta camisa blanca tiene una mancha negra: está sucia.

(35) Esta camisa llena de tinta negra tiene una parte limpia: *está limpia.

Diré que la suciedad es una tesis probable en el caso de (34), y la limpieza una tesis improbable en (35). Obsérvese de paso que por otra parte, 'tener una mancha negra' y 'tener una parte limpia' son argumentos para 'estar sucia (la camisa)' y 'estar limpia', como se puede comprobar en:

(36) Esta camisa blanca tiene una mancha negra, pero está limpia.

(37) Esta camisa llena de tinta negra tiene una parte limpia, pero está sucia.

Con lo cual queda patente que ' $p$ es argumento para $t$ ' no implica siempre 'de $p$ se puede sacar $t$ como conclusión'.

Consideremos ahora, suponiendo un contexto donde se trata de la puesta a punto de un nuevo prototipo de motor de coche:

(38) ?Ha habido un par de logros: el nuevo prototipo de motor está a punto.

(39) Ha habido un par de fracasos: el nuevo prototipo de motor no está a punto. 
Al igual que (35), (38) presenta una tesis improbable: unos cuantos logros no son del todo suficientes para concluir en la fiabilidad del nuevo prototipo. En (39) en cambio, un par de fracasos bastan para afirmar el carácter poco fiable de este mismo prototipo, tesis esta vez probable. Veamos ahora:

(40) A pesar de un par de logros, el nuevo prototipo de motor no está a punto.

(41) ?A pesar de un par de fracasos, el nuevo prototipo de motor está a punto.

Ejemplos donde podemos observar una distribución inversa a la que aparece en (38)/(39), fenómeno que se debe a la asimetría entre tesis probables y tesis improbables. De hecho, la tesis de la fiabilidad del prototipo es solamente improbable en (40), lo cual hace de la tesis opuesta 'no estar a punto' una tesis probable. Los fracasos hacen de 'no estar a punto' una tesis esta vez probable, siendo la tesis opuesta 'estar a punto' por tanto improbable. Podemos ahora interpretar las divergencias entre:

(42) A pesar de un par de logros, [on prétend + on admet] en los círculos autorizados que el nuevo prototipo de motor no está a punto.

(43) A pesar de un par de fracasos, [on prétend $+{ }^{*}$ on admet] en los círculos autorizados que el nuevo prototipo de motor está a punto.

Apoyándonos en lo anteriormente dicho, vemos que en (42), los logros hacen del buen estado del nuevo prototipo una tesis improbable, y del mal estado una tesis probable. En (43), al contrario, el mal estado del motor es una tesis probable en vista de los varios fracasos experimentados, y el buen estado una tesis improbable. De donde deducimos que on prétend que se combina con todo tipo de tesis ${ }^{20}$, mientras que on admet que requiere terminantemente una tesis probable.

20 No obstante, parece ser que on prétend que se combina mejor con las tesis improbables que con las probables. Se dice y se rumorea se comportan como on prétend en los ejemplos (42) y (43), pero no se comenta, que aparentemente no puede introducir una conclusión. 


\subsection{Interacción del locutor y del OMNI-locutor.}

Volvamos ahora al tema del OMNI-locutor y de la comunidad lingüística que conlleva.

a) On sait que versus on dit que:

Hemos visto ya que tanto on dit que $p$ como on sait que $p$ introducen un OMNI-locutor que afirma la veracidad de $p$, siendo la cuestión la opinión del locutor $L$ sobre el tema. Consideremos entonces:

(44)[On dit $+{ }^{*}$ on sait] que Cristóbal Colón era genovés, pero no (me lo creo + no estoy de acuerdo con este cuento).

(45)[On dit + * on sait] que hay un recalentamiento del planeta, pero para mí no es más que un rumor.

(46)[On dit + on sait] que Cristóbal Colón era genovés, opinión que comparto totalmente.

(47)[On dit + on sait] que hay un recalentamiento del planeta, de lo que estoy plenamente convencido.

Lo cual demuestra que $L$ es parte del OMNI-locutor en el caso de on sait que, pero no forzosamente en el caso de on dit que. No es de extrañar: sabido es que on sait que $p$ tiene $p$ como presupuesto, y que el presupuesto representa el punto de vista de una comunidad lingüística a la que $L$ forzosamente pertenece.

b) On raconte que, on admet que.

En estos dos casos, $L$ puede formar parte de la comunidad lingüística señalada por el OMNI-locutor:

(48) [On raconte + on admet] que el Gobierno cometió un grave error táctico, con lo cual estoy totalmente de acuerdo.

Pero sólo en el caso de on raconte que puede $L$ distanciarse del OMNI-locutor:

(49) [On raconte + ?on admet] que el Gobierno cometió un grave error táctico, pero no estoy en absoluto de acuerdo con esta opinión. 
En realidad, es posible una leve discrepancia de parte de $L$, probablemente debida al hecho que on admet que remite a un saber parcialmente fundado y que por tanto $L$ difícilmente puede pasar por alto:

(50) [On raconte + on admet] generalmente que el Gobierno cometió un grave error táctico, pero personalmente me queda la duda.

Donde dije digo digo Diego. Este último ejemplo es un claro caso de "esquizofrenia" discursiva: el locutor $L$ se parte en dos entidades enunciativas: el locutor en tanto que parte del OMNI-locutor, y el locutor en tanto que individualidad, teniendo estos personajes discursivos puntos de vista opuestos sobre el tema evocado.

c) On prétend que:

El caso más complicado y por tanto más interesante es on prétend que. Obsérvese primero que $L$ puede no compartir la opinión del OMNI-locutor que es el caso que $p$, como ilustrado en:

(51) [On prétend] que el Ministro de Hacienda quiere bajar los impuestos, pero no me lo creo, ni borracho de agua.

No solamente $L$ puede discrepar del OMNI-locutor, sino que con on prétend que es imposible que el locutor pueda compartir el punto de vista del OMNI-locutor. Se puede comprobar a través de la cominación del enunciado con los prefijos creo en lo que/no creo en lo que:

(52) No creo en lo que [on prétend + on raconte], que el Ministro de Hacienda quiere bajar los impuestos.

(53) Creo de veras en lo que [?on prétend + on raconte], que el Ministro de Hacienda quiere bajar los impuestos.

Para llevar a cabo el razonamiento, recurriremos a un análisis en contenidos presupuestos y puestos. Los criterios usuales demuestran que un enunciado [X prétend que $p$ ] tiene como presupuesto $p$ es falso. Por otra parte, sabemos que el presupuesto es el punto de vista de un OMNI-locutor - llamémoslo OMNI-locutor ${ }_{1}$ - que incluye forzosamente al locutor $L$. Con lo cual explicamos el contraste entre (52) y (53). El puesto de [X prétend que $p]$ es $X$ dice que $p$ es 
cierto, y en el caso que nos ocupa, será el puesto de otro OMNI-locutor, llamémoslo OMNI-locutor ${ }_{2}$ ya que $X=$ on. (52) y (53) tienen entonces como presupuesto $p$ es falso, de locutor OMNI-locutor ${ }_{1}$ siendo $L$ parte de este OMNI-locutor ${ }_{1}$. (52) tiene como contenido puesto L no está de acuerdo con el OMNI-locutor ${ }_{2}$ ('p es cierto'), compatible con el presupuesto $p$ es falso (de locutor el OMNI-locutor ${ }_{1}$ que incluye a $L$ ). En el caso de (53), el contenido puesto sería esta vez algo como L está de acuerdo con el OMNI-locutor ${ }_{2}$ ('p es cierto'), incompatible con el mismo presupuesto $p$ es falso (de locutor el OMNI-locutor ${ }_{1}$ que incluye a $L$ ). El ejemplo similar:

(54) [On prétend] que la contaminación es un fenómeno irreversible.

se analiza entonces de la manera siguiente: una comunidad lingüística incluyendo al locutor $L$ dice que la contaminación no es un fenómeno irreversible (contenido presupuesto). Otra comunidad asevera que la contaminación sí es un fenómeno irreversible. Ya que este último contenido es el puesto, $L$ no está forzosamente incluído en esta comunidad, a menos que ello se haga explícito. Lo que se puede comprobar en los siguientes encadenamientos:

(55) [On prétend] que la contaminación es un fenómeno irreversible, (y para mí, *es cierto + pero para mí, no es cierto $)^{21}$.

L no puede afirmar es cierto, porque de hacerlo iría en contra de sus propios presupuestos, lo cual sabemos es imposible.

\section{Marcadores genéricos y formas sapienciales.}

Uno de los motivos por los que nos interesan los marcadores mediativos es que pueden aplicarse al campo de la paremiología, de las formas sapienciales, como ya se ha sugerido en el apartado

21 El marcador discursivo para mí suele señalar el punto de vista que $L$ adopta e intenta imponer. Da lugar a contrastes como: Esta casucha, la levanté yo, con mis manos, y (para mí + ${ }^{*}$ en mi opinión), es un castillo, que se puede comparar con: - ¿Quées esto? - (Para mí + en mi opinión), es pescado. Véase Coltier-Dendale, op. cit. 
2.3. Obviamente, estos marcadores pueden remitir a un enunciador específico o bien indeterminado, y tener varias formas. Utilizaré aquí las formas de tipo como dice $X$. $X$ puede ser un autor concreto (como dice Lope de Vega), o un autor colectivo y anónimo (un OMNI-locutor): como se suele decir, como dicen, como se dice, como dice la sabiduría popular, etc. La posible combinación con un marcador mediativo específico nos proporcionará las sentencias, máximas, y apotegmas, como en:

(56) Como dice Machado, caminante, no hay camino, se hace camino al andar.

Y la posible combinación con un marcador esta vez genérico proporcionará las formas sapienciales anónimas, a saber, refranes, adagios, tautologías, dichos, etc. He aquí una muestra de la combinación de formas sapienciales con como se suele decir:

(57) Como se suele decir, en todas partes, se cuecen habas.

Como se suele decir, donde estuvieres, haz lo que vieres.

Como se suele decir, las apariencias engañan.

Como se suele decir, ha llovido desde entonces.

Como se suele decir, hay moros en la costa.

Como se suele decir, a otra cosa mariposa.

Como se suele decir, algo es algo.

Como se suele decir, el pasado, pasado está.

Como se suele decir, un día es un día.

Huelga decir que todas estas formas no son del todo equivalentes, y que pronto se distinguen varias subclases, entre las cuales las tres que figuran aquí: refranes, frases situacionales, y tautologías ${ }^{22}$. Nos interesaremos por las frases situacionales, como Hay moros en la costa. Tienen como características que no

22 Véase sobre el tema: Anscombre: 2000, 2006a, 2006c, 2010c. 
son genéricas como lo son por ejemplo En todas partes cuecen habas o Algo es algo: de hecho, remiten directamente a una situación que califican, y son combinables con indicaciones deícticas:

(58) Aquí, hay gato encerrado.

(59) ??Aquí, algo es algo.

(60) *Aquí, una golondrina no hace verano.

Tienen otra característica, que aparece en el siguiente contraste:

(61) Como se suele decir, una golondrina no hace verano.

(62) Una golondrina no hace verano, como se suele decir.

(63) Como se suele decir, algo es algo.

(64) Algo es algo, como se suele decir.

(65) Como se suele decir, hay moros en la costa.

(66) ??Hay moros en la costa, como se suele decir.

Además de proporcionar criterios para una posible clasificación de las paremias, los prefijos mediativos genéricos señalan una propiedad específica de las frases situacionales. Sin entrar en pormenores técnicos, no pueden ocupar una posición frontal temática, ya que califican una situación y por lo tanto forman parte del rema, siendo como se suele decir un marcador temático que sí puede ocupar la posición frontal. En cambio, las formas sapienciales como refranes y tautologías, que tienen todas las propiedades de un tema, pueden ir en posición frontal sin ningún inconveniente.

\section{Bibliografía}

Anscombre, J.C. (2000), «Parole proverbiale et structures métriques», Langages, 139, págs. 6-26.

Anscombre, J.C. (2005), «Le on-locuteur : une entité aux multiples visages «, in J. Bres, P. P. Haillet, S. Mellet, H. Nølke et L. Rosier (eds), Dialogisme et polyphonie : approches linguistiques, Bruxelles, De Boeck - Duculot, págs. 75-94. 
Anscombre, J.C. (2006a), "Polyphonie et classification des énoncés sentencieux. Les marqueurs médiatifs génériques», Le Français Moderne, 74, 1, págs. 87-99.

Anscombre, J.C. (2006b), «Stéréotypes, gnomicité et polyphonie: la voix de son maître», en Le sens et ses voix. Dialogisme et polyphonie en langue et en discours, L.Perrin (coord), Recherches linguistiques, 28, Univ. de Metz, págs. 349-378.

Anscombre, J.C. (2006c), «Las tautologías: características lingüísticas y funcionamiento", en Análisis del discurso: lengua, cultu$r a$, valores, M.Casado Velarde, R. González Ruiz \& $\mathrm{M}^{\mathrm{a}} \mathrm{V}$. Romero Gualda (eds), Arco Libros S.L., Madrid, págs. 1-13.

Anscombre, J.C. (2010a), «La polifonía: nociones y problemas», Archivum, LVIII-LIX, págs. 21-51.

Anscombre, J.C. (2010b), «A la croisée des chemins: la théorie des stéréotypes», in Etudes sur la combinatoire et la hiérarchie des composants, C. Alvarez Castro, Flor $\mathrm{M}^{\mathrm{a}}$ Bango de la Campa \& $\mathrm{M}^{\mathrm{a}}$ Luisa Donaire (eds), Peter Lang, Col. Sciences pour la communication, 90, Berne, págs. 7-23.

Anscombre, J.C. (2010c), «Las formas sentenciosas: un fenómeno lingüístico», Revista de Investigación Lingüística, 13, págs.1338.

Anscombre, J.C. (2013), «Polyphonie et représentations sémantiques: notions de base», in Opérateurs discursifs du français. Eléments de description sémantique et pragmatique, J.C.Anscombre, $\mathrm{M}^{\mathrm{a}}$ Luisa Donaire \& P. Haillet (eds), Col. Sciences pour la communication, Peter Lang SA, Berne, págs. 11-32.

Berrendonner, A. (1981), Eléments de pragmatique linguistique, Ed. de Minuit, París.

Casado Velarde, M. (1995), «El lenguaje de los medios de comunicación», en M. Seco \& G. Salvador (coords), La lengua española, hoy, Madrid, Fundación Juan March, págs.. 153-164. 
Coltier, D. \& Dendale, P. (2004), «La modalisation du discours de soi: éléments de description sémantique des expressions pour moi, selon moi, et à mon avis», Langue française, 142, págs. 41-57.

Donaire, Ma L. (2011), «La (non-) prise en charge, une dynamique polyphonique. Le cas de la stratégie concessive», en La prise en charge énonciative. Etudes théoriques et empiriques, Col. Champs linguistiques, De Boeck-Duculot, Bruxelles, págs. 55-74.

FERnÁNDEz Bernárdez, C. (2002), Expresiones metalingüísticas con el verbo decir, Monografías $n^{\circ} 97$, Universidade da Coruña.

HAILLET, P.P. (2002), Le conditionnel en français: une approche polyphonique, Col. L'essentiel, Ed. Ophrys, París.

Leeman, D. (1991), "ON thème", Linguisticae Investigationes, XV, 1, págs.101-113.

Martín Zorraquino, Ma.A. (1998), “Los marcadores del discurso desde el punto de vista gramatical", en $\mathrm{M}^{\mathrm{a}}$.A. Martín Zorraquino \& E. Montolíu Durán (coords), Arco/Libros S.L., Madrid, págs. 19-53.

Martín Zorraquino, Ma.A. \& Portolés Lázaro, J. (1998a), "Los marcadores del discurso", Gramática descriptiva de la lengua española, I.Bosque \& V.Demonte (coords), Ed. Espasa, Madrid, vol. 3, págs. 4051-4213.

Portolés LÁzaro, J. (1998a), Marcadores del discurso, Ed. Ariel S.A., Barcelona.

Portolés LÁzaro, J. (1998b), “La teoría de la argumentación en la lengua y los marcadores del discurso", en Mª.A. Martín Zorraquino \& E. Montolíu Durán (coords), Los marcadores del discurso. Teoría y análisis, Arco/Libros S.L., Madrid, págs. 71-91.

Jean-Claude Anscombre Directeur des Recherches

CNRS (LDI) 